Visualization and data analysis 2014 : 3 - 5 February 2013, San Francisco, California, United States ;

proceedings IS\&T/SPIE electronic imaging, science and technology/sponsored and publ. by IS\&T - the

Society for Imaging Science and Technology; SPIE. Pak Chung Wong ... (Ed.). - Bellingham, Wash. : SPIE

[u.a.], 2014. - 90170C [9017-11].- (Proceedings of SPIE ; 9017). - ISBN 978-0-8194-9934-9

\title{
Collaborative Data Analysis with Smart Tangible Devices
}

\author{
Johannes Fuchs ${ }^{a}$, Roman Rädle ${ }^{b}$, Dominik Sacha ${ }^{b}$, Fabian Fischer $^{b}$ and Andreas Stoffel ${ }^{b}$ \\ ${ }^{a, b}$ University of Konstanz, Germany
}

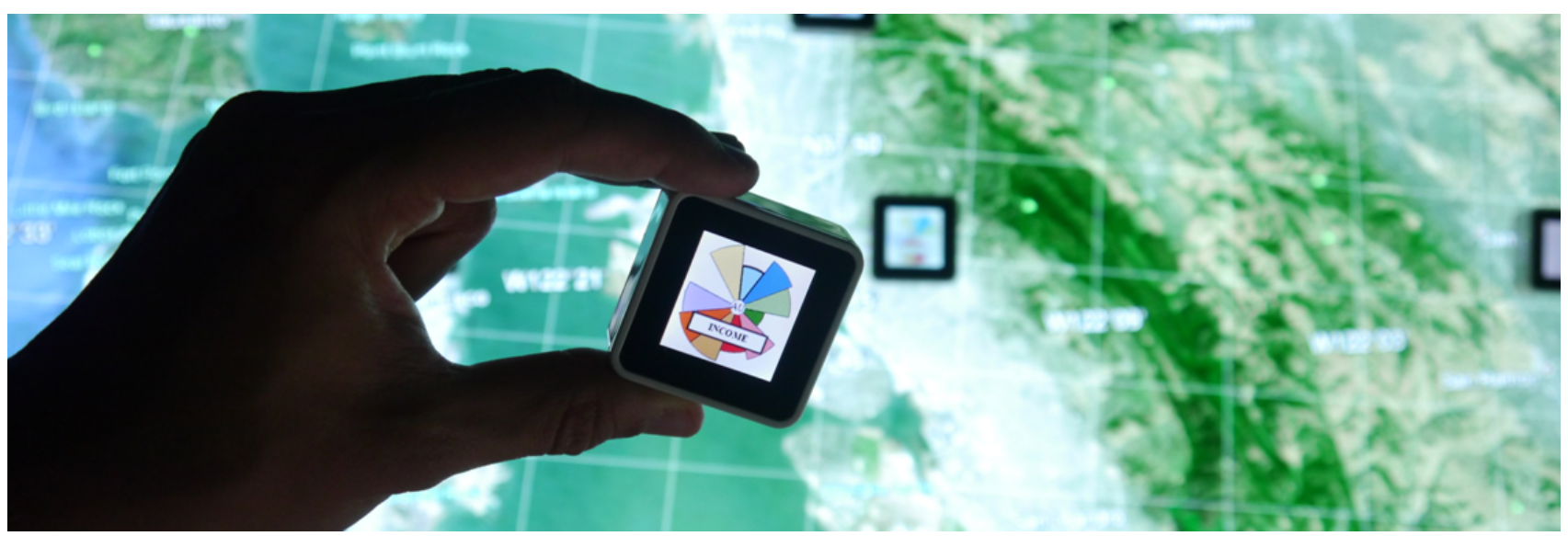

Figure 1. Tangible Data Analysis: The image shows in the background a multi-touch table visualizing a geographic map of the US east coast. A user is exploring census data with the system by using Sifteo Cubes as smart tangibles.

\begin{abstract}
We present a tangible approach for exploring and comparing multi-dimensional data points collaboratively by combining Sifteo Cubes with glyph visualizations. Various interaction techniques like touching, shaking, moving or rotating the displays support the user in the analysis. Context dependent glyph-like visualization techniques make best use of the available screen space and cube arrangements. As a first proof of concept we apply our approach to real multi-dimensional datasets and show with a coherent use case how our techniques can facilitate the exploration and comparison of data points. Finally, further research directions are shown when combining Sifteo Cubes with glyphs and additional context information provided by multi-touch tables.
\end{abstract}

Keywords: Sifteo Cube, tangible, data analysis, visualization, interaction, glyph, public space

\section{INTRODUCTION}

Visual analysis of data is getting a mass phenomenon. Applications such as the OECD - Your Better Life Index ${ }^{1}$ are attracting the attention of many users, that are interested in data. This is supported by two recent developments. First, computers are getting more and more mobile and can be found everywhere. Touch and gesture interfaces allow users to interact with computers easily in a more natural way. The second development is supported by the ability to work with data detached from desktop computers. Data is moving more and more into public spaces, for instance, museums or libraries are experimenting with interactive visualizations to communicate information. Important in these scenarios is the ability to work with data and the analysis space in an intuitive way that also enables tightly-coupled collaboration and loosely-coupled parallel work.

Whereas existing data analysis interfaces are tailored for expert users, these interfaces are not really useful in public spaces. Expert users need many opportunities to analyze the data and have different views from different perspectives. As consequence, interfaces for experts ask too much from an occasional user. In public spaces, the situation is different. Users may enter or leave at any time, they may want to exchange information and findings

\footnotetext{
Further author information: (Send correspondence to Johannes Fuchs)

a fuchs@dbvis.inf.uni-konstanz.de

${ }^{b}$ \{roman.raedle,dominik.sacha,fabian.fischer,andreas.stoffel\}@uni-konstanz.de
} 
with each other; or request for help with a particular analysis task. If the interface is not able to allow easy interaction with the data and visualizations, users loose interest in further use of the analysis interface. Wigdor and Wixon ${ }^{2}$ have shown that in such environments more natural user interfaces and interaction paradigms should be preferred. This is one reason, why in public spaces the classical WIMP (Window, Icons, Menus, Pointer) interface is replaced with more natural interaction, for instance, touchable displays in mobile phones or handhelds, or speech, motion and gesture controls.

Natural data analysis interfaces are especially important for public spaces, where different users come together and analyze data. An interface on smart tangible objects is one solution for the arising problems in this scenario. With tangible objects, users can interact with data and visualization to select and filter relevant data or choose parameters by interacting with physical objects. They can also be used for mixed focus collaboration, which is a common problem in group work ${ }^{3}$ For instance, different users with different needs and interests are coming together to analyze a data set. When someone finds something interesting, the insight might be exchanged with other researchers looking at different aspects of the data. The interesting data or finding can be exchanged between users by exchanging the tangible objects representing the interesting fact. In addition, tangible objects support an external and physical arrangement of data points, which helps user to create mental maps of the data or analysis results $\frac{4}{4}$ Another important aspect of tangibles is the similarity with natural working environments. Users can move, remove, or group tangibles and thereby the associated data as they are natural objects. This interaction builds upon users' pre-existing knowledge and skills of everyday life and, therefore, may reduce their mental efforts $\frac{5}{5}$

In tangible user interfaces, the tangible objects are usually simple physical objects with very limited interaction capabilities. An alternative is offered with Sifteo Cubes, which are an award-winning interactive gaming platform..$^{6}$ The product is the commercial outcome of a research idea called Siftables. ${ }^{7}$ After iteratively improving Siftables the creators came up with a small tactile entertainment system. The idea behind Sifteo Cubes is to offer a variety of games making use of a gestural interface for problem solving. The device has a touch sensitive low resolution display ( $128 \times 128$ pixels) and offers different interaction possibilities. With a three-axis accelerometer the cubes can detect spacial movement (e.g., tilting, rotating, or shaking). Additionally, the touch sensitive screen recognizes a single touch event (e.g., a press). To detect neighboring cubes each cube contains proximity sensors and a $2.4 \mathrm{GHz}$ wireless radio to communicate with the Sifteo Base. Up to twelve cubes can be connected at the same time.

We present a smart tangible interface design based on Sifteo Cubes for data analysis in public spaces, such as museums or libraries. The advantage of the Sifteo Cubes compared to other tangibles is the ability to dynamically change the information shown on the cube, the ability to interact with the cube, and the possibility to arrange the cubes in a grid. We systematically designed a tangible interface based on Sifteo Cubes for interactive data analysis by making use of these possibilities. Our interface is designed for collaborative data analysis in multi-user environments. We demonstrate our technique with a use case and show the applicability and usefulness of our approach. Finally, we discuss the advantages and disadvantages, and give an outlook for further improvements and research.

\section{RELATED WORK}

Our work here draws upon existing research that explore compact visualizations and novel navigation techniques utilizing tangible physical objects for data exploration and object manipulation. The related work is split into two parts. The first part provides an overview on related work that concentrates on glyph visualizations. The second part of the related work summarizes those projects that focus on interaction.

\subsection{Visualization}

Sifteo Cubes only offer a limited resolution and size of the screen. This constrains the design space and restricts the search for related work to very compact visual representations for data points. Glyphs or iconic displays use space in an efficient way and are, therefore, a convenient visualization method. However, due to the many possibilities of how to map data to visual variables, there exists a nearly endless amount of glyph designs. To briefly structure this related work section we distinguish between designs using position or direction to layout the dimensions and the visual variables used for encoding the data value. 
Position. A famous example for positioning the dimensions linearly are small bar charts called profile glyphs 89 They have a common baseline and visualize the data values with length enabling an easy value comparison. Same thing is true for dot plot glyphs, $\frac{10}{10}$ line glyphs (e.g., sparklines $\frac{11}{11}$ ), or gestaltlines,$\frac{12}{12}$ however, they use vertical position or orientation instead of length to represent the data value. Depending on the number of dimensions these designs profit from a more rectangular like aspect ratio to have enough space visualizing the single dimensions.

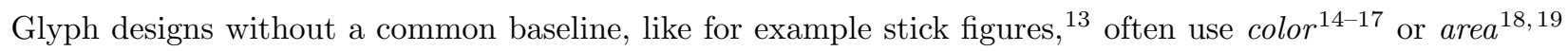
to represent the data value. For comparing quantitative data these designs are not as accurate as simple length encodings with a common baseline ${ }^{20}$ Of course, other designs do not necessarily map the data value to only one common visual variable. The well-known chernoff faces ${ }^{21}$ make use of different face characteristics (e.g., size of the nose, orientation of the mouth, etc.) to represent data values. However, the designer has to carefully think, which dimension should be visualized by which visual face characteristic. Some are more dominant than others, which could influence the perception of the data point.

Direction. On the other hand, there are designs, which use a circular layout to arrange dimensions. These designs usually have a quadratic aspect ratio to not introduce a bias towards certain dimensions. The well-known star glyph,$\frac{22}{22}$ for example, positions the dimensions in a radial fashion and uses a length encoding to represent the data value. By connecting the endpoints of the lines, the star glyph creates an overall shape. Furthermore,

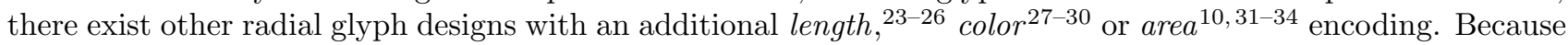
of the missing baseline, these designs do not perform as good as linear designs with a common baseline ${ }^{35}$

This summary of different glyph designs does not claim to be exhaustive. For a more complete list of iconic representations see the survey of Ward 36 or Borgo $\stackrel{37}{37}$ However, we want to show that there is a nearly endless amount of possibilities of how to represent data using glyphs. In order to find a suitable representation designers have to really think about the way they want to visualize the data. Therefore, we have carefully build a design space to investigate possible visualizations for Sifteo Cubes in Section 3.1 .

\subsection{Tangible Interaction}

In this section we provide an overview of related work that makes use of physical objects to manipulate a virtual information space. The work is divided in two categories: passive and active tangibles. The difference between both is that active tangibles are equiped with additional electronic components such as IR sensors to measure proximity or displays for visual output.

Passive Tangibles. Already in 1995, Fitzmaurice et al ${ }^{38}$ coined the term graspable user interfaces, which is a paradigm that extends virtual objects to the physical space and intermixes virtual and physical interaction. This allows control and direct manipulation of virtual objects through bricks (or nowadays known as tangibles). The authors proposed basic interaction concepts for the bricks like lift up (detach) and put on (attach) or move and rotate. The interaction space, however, is limited because bricks are passive physical blocks that need to be put on a sensing surface.

Nowadays, most interactive tabletops that offer such a rich interaction do only provide a single tangible layer,

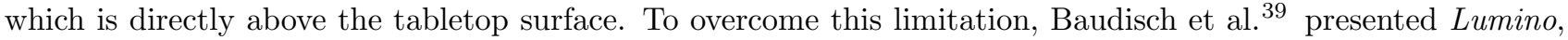
a system of building blocks for tabletop interaction. With their approach, the interaction can be extended with $3 \mathrm{D}$ arrangements of tangibles. However, the system requires an interactive hybrid surface and since Luminos are passive building blocks they do not offer interaction such as shaking or tilting. Another drawback of tangible interaction on interactive hybrid surfaces is that they are restricted to horizontal layout because of naïve physics when a user releases a tangible. Two techniques bypass this issue. The first is Geckos ${ }^{401}$ by utilizing a magnetic surface that is able to sense marker arrangements and thus recognizes identities, positions, and orientations of vertically placed tangibles. The second project Vertibles ${ }^{41}$ uses an adhesive film that is put underneath the physical object. It makes use of microscopic suction cups and allows to put so called Vertibles on vertically mounted surfaces. Although aforementioned tangible user interfaces offer a rich set of interaction possibilites and unlike the Sifteo Cubes, the tangibles themselves cannot display additional and dynamic content. 
The DataTiles by Rekimoto et al., ${ }^{42}$ though, provide flexibel configurations of views with dynamic data and actions on them through transparent tiles. Nearby arrangements of tiles express relations and connects views and corresponding actions, e.g., a view for a floor plan is updated by changing paramenters of a query that is expressed on a tile right next to the view tile. With this, window managment becomes an entire physical activity. Spindler et al ${ }^{43}$ take this to the third dimension and combine the approach of spatially-aware displays with the concept of a magic lens. Their Tangible Views extend a multi-touch tabletop to the third dimension, so that users can apply familiar physical interaction to navigate and explore in complex information spaces. Small paper-based passive displays are used as lenses to investigate different kinds of visualizations in more detail. The plates can be moved, rotated or flipped to offer several interaction possibilities. In contrast to Sifteo Cubes these displays need additional and expensive hardware such as projectors and motion capture cameras, limiting their application area to a certain predefined location.

Active Tangibles. The Stackables ${ }^{44}$ are a tangible approach to facilitate the facetted information seeking process. Small input devices with displays are stacked vertically to query a database. Each Stackable device filters the underlying dataset according to a set facet and multiple facet labels. The filter sets are combined with a Boolean $A N D$ if the devices are stacked. The final result of such a query is, then, displayed on an additional screen. Unlike Sifteo Cubes, Stackables are limited to filtering tasks with only a limited set of interaction possibilities.

Zigelbaum et al ${ }^{45}$ introduce an approach to enable a collaborative and non-linear film editing by physically arranging active tokens. This Tangible Video Editor enables stitching of multiple video sequences by assembling several small devices in line. It, moreover, allows to attach several features such as sequence transitions. They studied the interaction and communication behaviours during a collaborative video creation task. In their observations they found that people first discuss different video storylines having seen a few clips while in the baseline condition with a traditional desktop video tool participants began their sessions watching all sequences first. Since the Sifteo Cubes offer a similar flexibility, we hope to foster such early discussions during collaborative analysis of multi-dimensional data points.

\section{CONCEPT}

The major difference between Sifteo Cubes and classic WIMP based visualizations is the fact, that the cubes combine the visual aspect (i.e., the display) and the interaction possibilities within a single hardware component. Therefore, the user is able to physically take a data point in his hands and to a different location/position. This action is known from real world interaction with everyday life objects and emphasizes the idea of having a natural user interface (NUI). Sorting or reordering interactions can be done naturally by the user. He does not need to understand any implementation details because the whole exploration process is intuitive. The user can group data items on the table by simply placing them next to each other or he can get rid of them by wiping the data points from the table. A demo video of our interaction concept can be found at http: //www.youtube.com/watch?v=yrPqqa4CNaY, It is like playing with bricks!

The user may start his exploration in an initial overview grid setting where the cubes are meant to present a rough overview over the data space. One single cube either represents one data point or a dimension. The positioning of the single cubes on a surface influences the analysis of the user. It is easier to compare cubes, which are closer together than far distant cubes. However, the flexibility of the small displays allows a fast rearrangement of the initial setting. The analyst may gain some further insights while interacting with the cubes. Tightly connected to the repositioning of data points is the selection of single elements. After detecting points of interest, the user can take these single elements and isolate them from the initial arrangement to reduce the amount of distractors surrounding this data point. By separating interesting data points, the user applies a filtering step to the data tailored according to his or her knowledge.

We split up the following content in two parts. First, the visualization representation of the Sifteo Cube and, second, the interaction concept for the data analysis. In the visualization representation section we justify our display design. Due to the limited space and resolution restrictions of the Sifteo Cubes we developed compact representations (i.e., glyphs) to visualize data. The interaction part covers the semantics of several interaction 


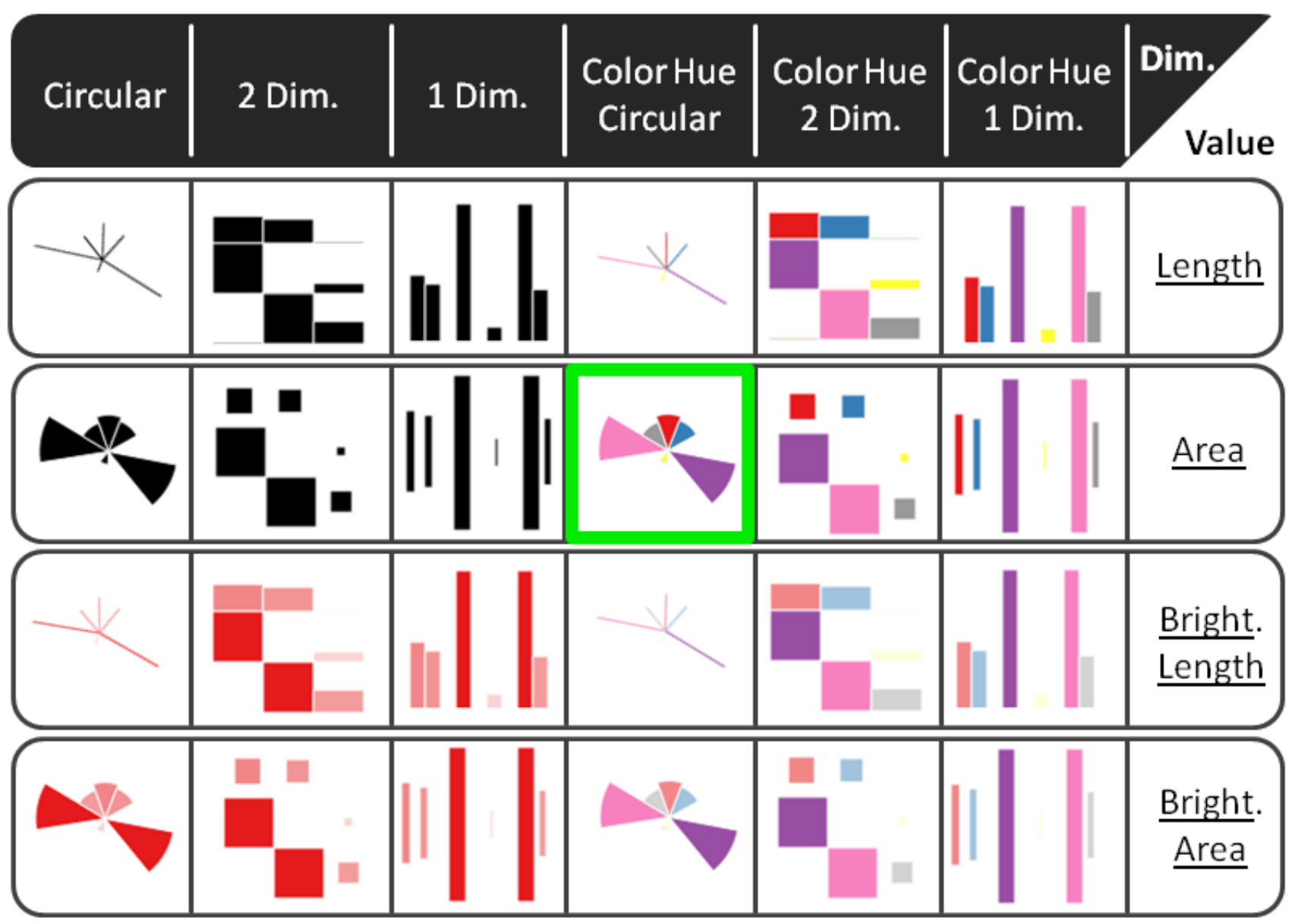

Figure 2. Design Space: Illustration of the glyph design space for Sifteo Cubes. The y-axis represents different visual variables for the value encoding, the x-axis represents different strategies for visualizing the data dimensions. The glyph used in our applications is highlighted.

techniques the user may perform in order to explore the data. The interaction techniques depend on the number of involved cubes, their constellation and the performed operations (e.g., rotating, touching or shaking).

\subsection{Visual Representation}

The tiny display and the low resolution of the screen does not allow for complex visualizations. That is why we focus on the development of a compact glyph representation to visualize data. Glyphs have the advantage of communicating many aspects of the data without using too much space. In the following we will discuss the design decisions made while creating an appropriate visual representation.

According to the type of data, different visual variables have to be chosen to create the overall glyph design. For nominal data a check for equality is sufficient. Common visual variables for this kind of data are shape, color (hue) and texture ${ }^{46}$ For ordinal data, however, an additional visual ordering must be possible, too. Therefore, the visual representation has to communicate a natural order. Due to the difficulty of comparing distances with saturation, brightness, orientation, blur, sketchiness, dashing or motion, these kinds of visual variables with a natural order are an appropriate choice. The highest demand for the visual representation puts the numeric data type because this kind of data has a distance metric, which must be perceivable. Therefore, numeric dimensions must be visually comparable, orderable and their distances measurable. Possible encodings are size, brightness, saturation and vertical position! 47 


\subsubsection{Constraints}

Many glyph designs encode the data attributes with different visual variables (e.g., chernoff faces map data to visually different face characteristic 21 ). This mixture of visual cues introduces a bias towards certain dimensions. The most important dimensions are mapped to the most significant glyph characteristics. To avoid additional dimension ordering or sorting, we will not consider designs, which promote certain dimensions. For unknown independent data dimensions this constraint is mandatory.

To further reduce visual bias, we will only consider a many-to-one-mapping, where all attributes map to a common type of graphical attribute ${ }^{[36}$ Additionally, each dimension should be uniquely identifiable. The design must be able to communicate the whole information by the unique mapping of a visual variable to the data dimensions and to the data values for each dimension.

\subsubsection{Data Value}

When it comes to the overall glyph representation, designers have to distinguish between the visual variable for encoding the data value and the way of how the dimensions are displayed. As already explained size, brightness, saturation and vertical position are possible encodings for quantitative data.

Visual Variable: Size. Cleveland and McGill sub-divide size in length, area and volume ${ }^{20}$ Generally, length will be judged more accurately than area, however, according to Ware, area is capable of conveying larger variations ${ }^{47}$ In literature, both kinds of encodings are used 831 As a consequence, we will use both variables in our design space separatly. Volume, on the other hand, will be discarded because Ekman and Junge, and Ware state that volume is only poorly judged compared to length and area 474.48

Visual Variable: Brightness and Saturation. Brightness and Saturation will be considered as only one visual variable since they are very similar. Brightness is the change from color to white and saturation from color to gray. Since it is hardly possible to compare distances between data points using either saturation or brightness we will not include these visual variables in our design space separately, but in combination with others (i.e., as a double encoding).

Visual Variable: Vertical Position. Vertical position will not be considered as well because the position of single marks can hardly be perceived due to the minimal size of a glyph. Since glyphs are really compact representations of data points, the available space is very limited and confine the comparison of different located marks.

\subsubsection{Data Dimension}

Besides the appropriate encoding for the data value, the separate dimensions have to be displayed as well. The layout should not introduce any bias and must, therefore, be as regular as possible. We will consider a one dimensional linear positioning, a two dimensional arrangement and a circular layout. Using solely a color, shape or texture encoding for the dimensions is not feasible because without changing the position data points will be overplotted. Of course, some of the design decisions can be combined to achieve a double encoding for either the data value or the dimensions or a double encoding for both simultaneously.

In our opinion, radial designs profit most from a quadratic aspect ratio like preset by the Sifteo Cubes. To not support single dimensions, the circle is divided into slices of equal size according to the number of data attributes. The slices differ in color hue, each one encoding a single dimension. This double encoding (i.e., color hue and angle) helps the analyst to distinguish between the different attributes of the data point. We discard an additional brightness encoding, because of the low visual quality of the Sifteo Cubes. The value of each dimension is encoded by the area/length of the corresponding slices. The resulting glyph is highlighted in Figure 2 


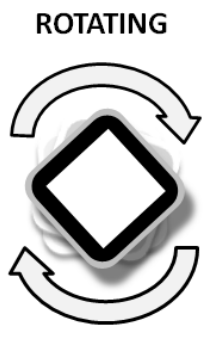

Select

Dimension

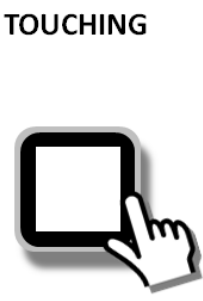

Use Case

Specific
TILTING

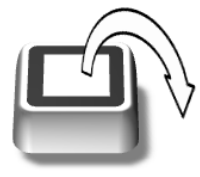

Switch

Interaction Mode
SHAKING

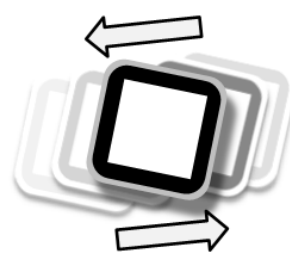

Switch

Screen Mode

Figure 3. Cube Interaction: Illustration of possible interaction techniques for a single Sifteo Cube.

\subsection{Interaction Techniques}

As a starting point we consider elementary tasks users may perform according to the task topology described by Andrienko and Andrienko $\frac{49}{6}$ We limit our system to the elementary lookup and comparison tasks. To create an interaction concept, which best supports users in analyzing data, we conducted a qualitative pre-study. The displays of the cubes have been labeled with the names of data points (e.g., A-1, B-1, A-2) and one with an example visualization of a data point. The participants where asked to perform different tasks, for instance comparison or grouping of data points, with the cubes. Five participants have been recruited from the local students population. Each participant had to perform the same basic analysis tasks. An experimenter was guiding the study and explained the setting, the data, and possible interaction techniques supported by the cubes. Figure 3 illustrates the basic interactions, which are namely rotating, touching, tilting, shaking, and moving. This information was also printed on a sheet, which was visible all the time to make sure the students remember everything. Participants were told to think aloud while solving the tasks, so a second experimenter was able to take notes about their thoughts and their behavior.

The results show a consistent user behavior over all tasks. In the first task, the participants had to group the smart tangibles according to the number displayed on the label. All participants first scanned the whole set of tangibles and grouped them spatially, having a short distance between data points within a group and larger distances between different groups (Figure 4). Additionally, most of the participants connected the cubes within the groups, as well. For the second task, all cubes showing the letter "A" had to be deleted. The participants took the corresponding cubes and placed them far away from the others out of the focus. One student additionally turned the screen towards the table so the display was not visible anymore. For task three the experimenter took the cube with the example visualization and told the participants that one dimension (i.e., one slice) is selected and they have to select a different dimension. After scanning through the different possible interaction techniques, the participants took the cube and rotated it. Only one student tried to touch the dimension in order to select it. However, the experimenter reminded the user that the device does not recognize different areas of the touch event, so he changed his initial approach and started to rotate the cube, as well. Task four was about the comparison of two or more data points. The students directly connected the cubes, which have to be compared, but the arrangements differ. With higher amounts of cubes, the students were not sure whether to connect them linearly or in a matrix layout. For comparing two different sets of cubes, the participants first grouped them and then connected the cubes within each group. In the last task participants were told to display further details about the cube with the example visualization. All participants took two or more cubes to create an additional screen for displaying details, but the students followed different approaches. One student touched the cube to signalize the others to show details, another student tilted the cube so the details "flow" to the other cubes, two other participants shook different cubes to get a detailed screen, and the last student even stacked two cubes vertically. Obviously, this was the toughest task for the participants with very diverse solutions.

In the following we used these findings to create a solid interaction concept for smart tangible devices. Since all users agreed on the same interaction paradigms for the comparison and dimension selection tasks, we adopted their approach. For exploring data points in detail, the participants used different interactions. We decided to introduce special exploration cubes, providing the users with additional screen space. 


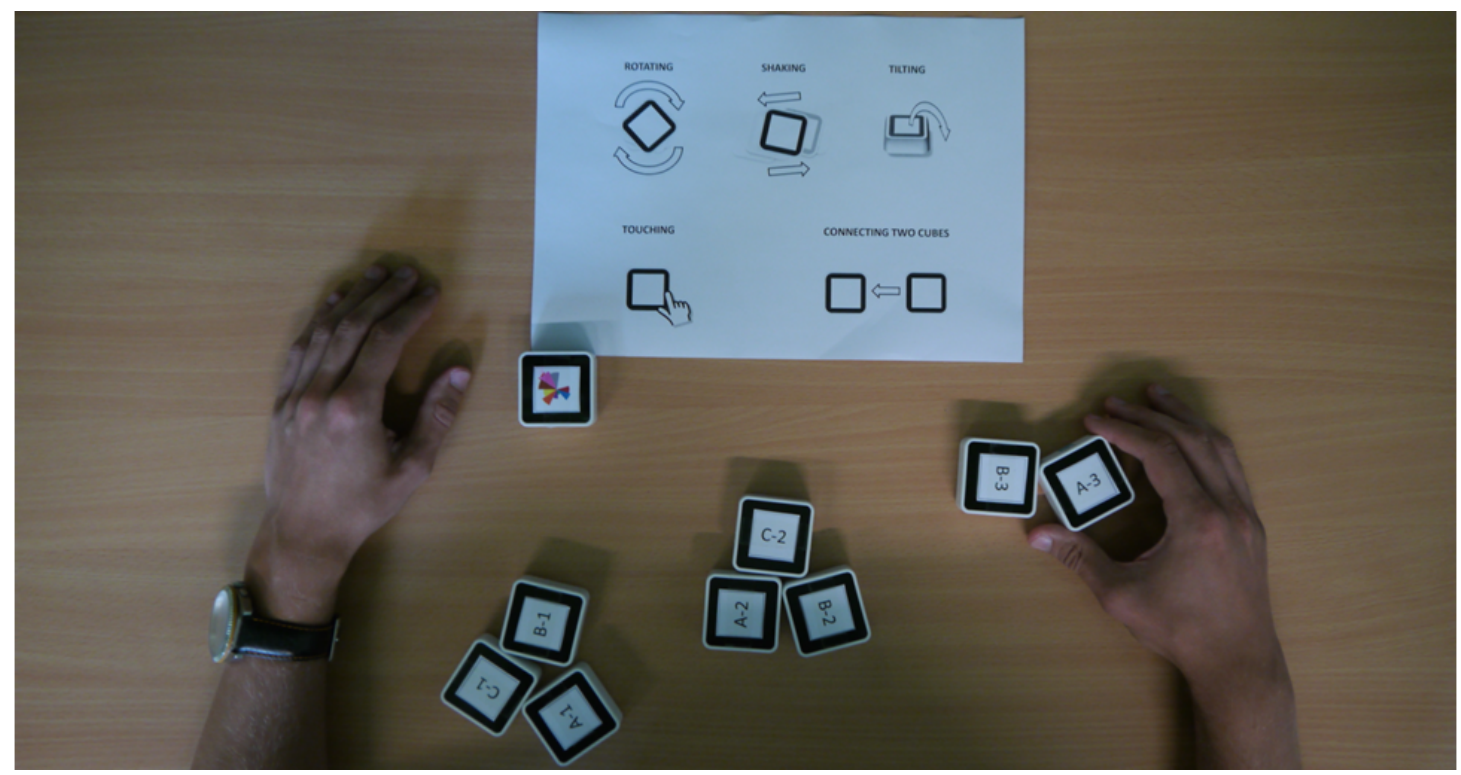

Figure 4. User Study: Participants had to perform basic analysis tasks using smart tangibles (i.e., grouping data points). The displays have been covered with unique IDs except for one showing an example data point. A sheet on the table shows the interaction techniques supported by the tangibles.

In the following we formalize our interaction concept. First, we define five possible states for the cubes. In Data state (D) a cube is associated with a data point and showing a representative glyph. Cubes in Comparison state $(\mathrm{C})$ are used to compare two data points with each other. The glyph representation is in this case replaced with a bar chart allowing easy comparison of the data points. Cubes can be switched into Exploration mode (E), serving as additional space for exploration. The combination of exploration cubes with data or comparison cubes leads to two more states called Extended Data (ED) and Extended Comparison (EC). We use these states to describe our interaction concepts below. The $\odot$ operator represents a horizontal or vertical neighborship of cubes. Other arrangements of cubes are not considered. The possible combination of two cubes are shown in Figure 5 and of three cubes in Figure 6 .

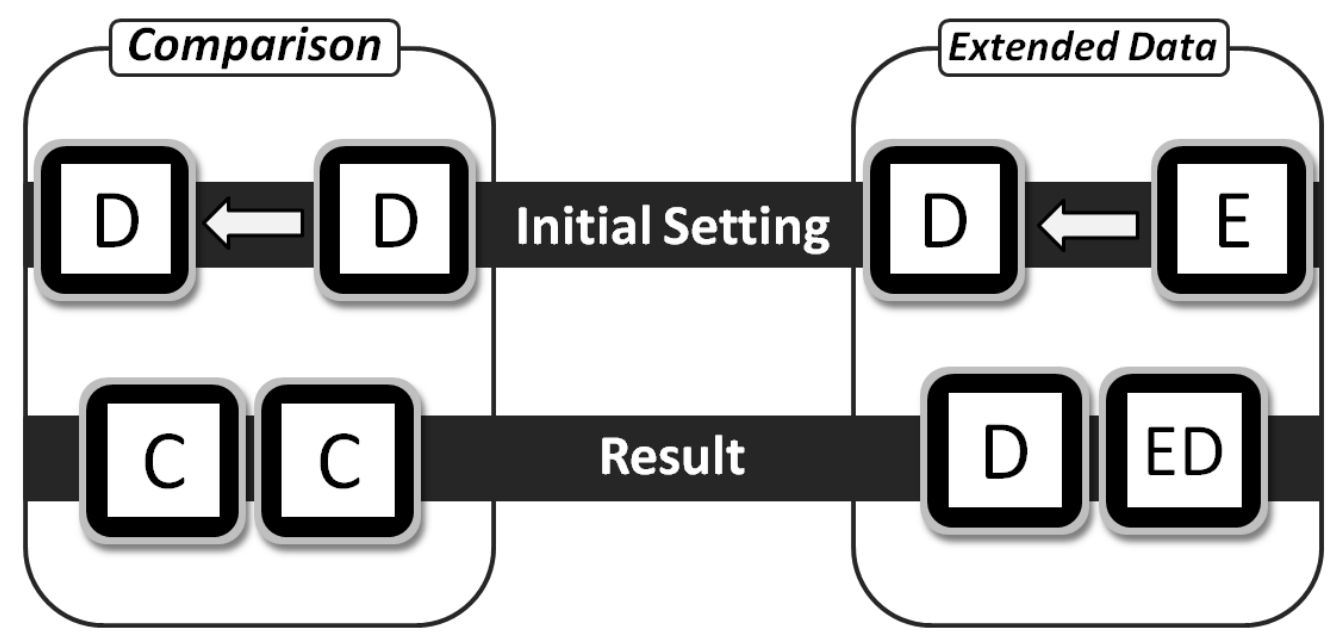

Figure 5. Connection: Two Sifteo Cubes can be connected enabling additional exploration possibilities. A comparison of two data points can be achieved by connecting two data cubes (see left side). The space for visualizing a single data cube can be extended with an additional exploration cube (see right side). 


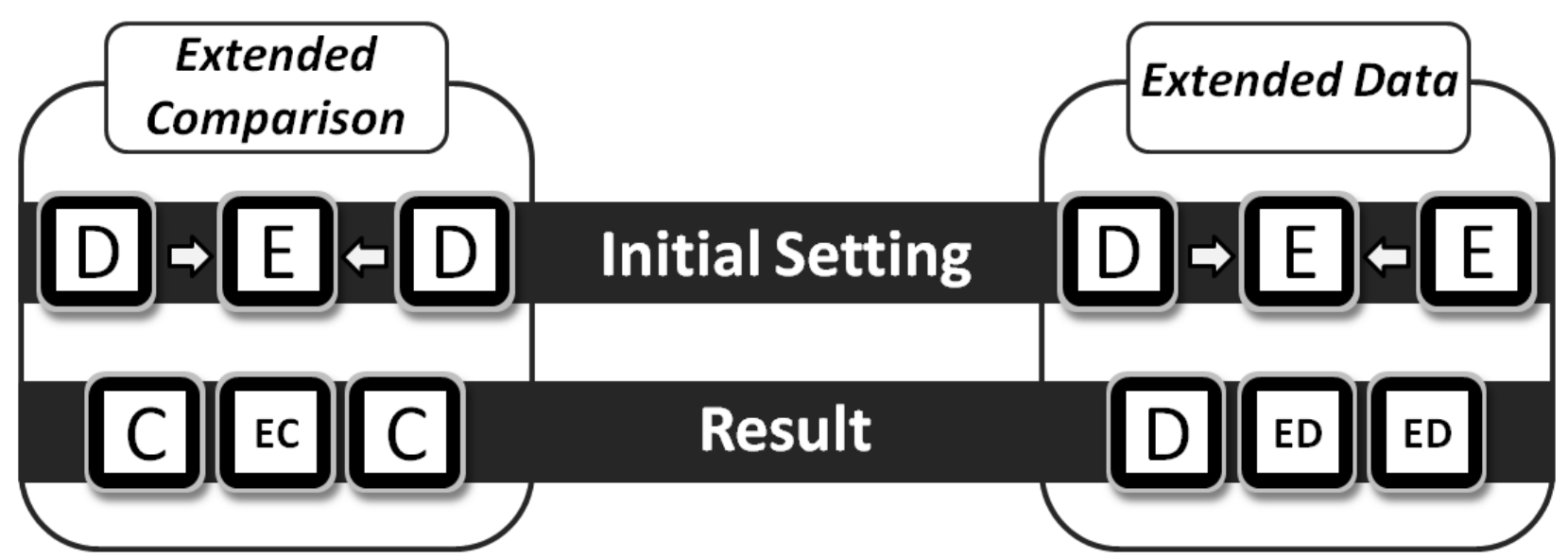

Figure 6. Connecting Multiple Cubes: Several Sifteo Cubes can be connected to enable more complex states. For a more detailed comparison of two data points one or more exploration cubes can be put between two data cubes (see left side). Gaining space for visualizing data points is possible by enlarging the extended data state with additional exploration cubes (see right side).

\subsubsection{Basic Cube Interactions}

Each cube is either in data state (D) or exploration state (E). By shaking a cube its state can be switch; a data cube becomes an exploration cube and vice versa. A single dimension of the data can be highlighted. To change the highlighted dimension, the user has to take a data cube tilt it towards her- or himself and rotate the cube. The highlighted dimension is switching through. If the desired dimension is highlighted, the cube can be put back onto the table.

\subsubsection{Comparison}

To compare different data points, the according cubes must be put together. Equation 1 shows the basic formalization of a comparison of data cubes. By putting two or more data cubes (D) together, the cubes are switching their mode to the comparison state (C). The glyphs shown on the cubes representing data points are not useful for comparing data, because of the chosen radial glyph. In comparison state the glyphs are therefore replaced by bar charts, allowing a visual comparison of the data points. For comparing single dimensions, the length encoding of bar charts is more suitable than the area encoding of Nightingale representations. ${ }^{32}$ Due to limited visualization space of the cubes, only the difference between the data points associated with the cubes are shown. The bar of each dimension is oriented towards the cube with the higher data value. The length of the bar is representing the difference.

$$
D_{1} \odot D_{2} \odot \ldots \odot D_{n} \Rightarrow C_{1} \odot C_{2} \odot \ldots \odot C_{n}
$$

\subsubsection{Extended Data}

A data cube shows a glyph representation of a single data point. In order to visualize more details, the data cube must be combined with one or multiple exploration cubes, as shown in Equation 2 In this case the exploration cubes become extended data cubes. The original data cube remains unchanged and still shows the glyph representation of the data point. The extended data cubes are now used to visualize more details about the data point in tabular form.

$$
D \odot E_{1} \odot E_{2} \odot \ldots \odot E_{n} \Rightarrow D \odot E D_{1} \odot E D_{2} \odot \ldots \odot E D_{n}
$$




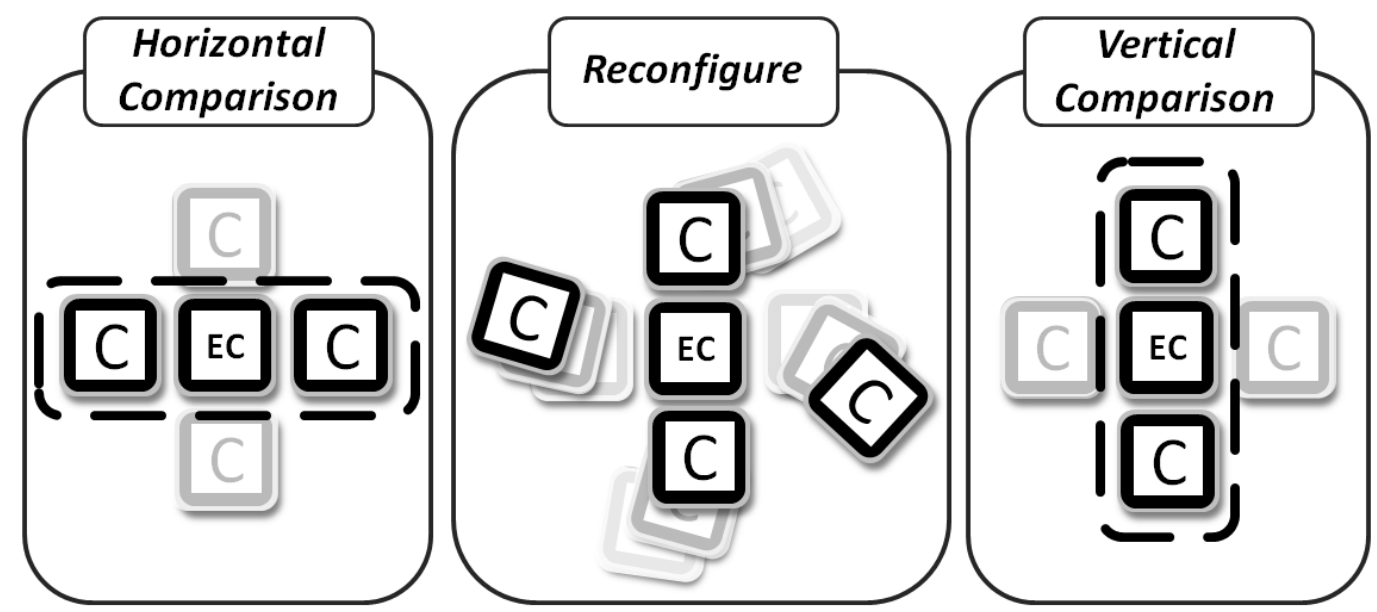

Figure 7. Example: Not all horizontal or vertical arrangement of cubes are allowed. For instance, the extended comparison in vertical and horizontal direction over one exploration cube is not supported. In this case some cubes have to be removed in order to change the comparison direction.

\subsubsection{Extended Comparison}

Similar to the extension of a data point, exploration cubes can be used to show more details of a data point comparison, as shown in Equation 3 . One or more exploration cubes are put between two data cubes. The exploration cubes are then switching to the extended comparison mode (EC) and showing more details about the comparison of the left and right comparison cube in tabular form.

$$
D_{1} \odot E_{1} \odot E_{2} \odot \ldots \odot E_{n} \odot D_{2} \Rightarrow C_{1} \odot E C_{1} \odot E C_{2} \odot \ldots \odot E C_{n} \odot C_{2}
$$

\subsubsection{Restrictions}

The cubes can be arranged either horizontally or vertically. Crossing directions are only allowed with data or comparison cubes. Extending a data cube with exploration cubes in multiple directions simply doubles the extended information. A data cube can be compared with up to four neighbors by using all vertical and horizontal directions for comparison. Crossings on exploration cubes are not allowed, because the exploration cube can only show information for one direction. Due to the limited visualization space of the cube, splitting is not possible.

In case a cube is part of an invalid arrangement, the cube shows an error message. Consequently, the user has to reconfigure the arrangement of cubes to get back into valid configuration. For instance, the reconfiguration to change a horizontal into a vertical comparison is shown in Figure 7.

\section{USE CASE}

In our concrete scenario, a school class in politics is visiting a library to get further information about country characteristics, specifically about migration. The library offers aside from books, magazines, and computers also interactive multi-user visual interfaces (e.g. multi-touch tables) to access public databases. In detail, one of these interfaces offers a multi-touch table and additional smart-tangibles. Some students are immediately attracted by the novel installation. They start playing around with the multi-touch interface and managed to load the migration database. As a consequence, the multi-touch table shows a political map of all counties in the US. On top of each county a glyph represents the underlying migration data. A librarian notices the interest of the students in the installation. He recognizes that too many students try to interact with the table. The available space on the table is not sufficient to allow all interested students to work simultaneously with the data. So he offers them the possibility to use a new research prototype for collaborative data analysis. This prototype is based on our smart tangibles concept (Figure 8).

With our interface multiple users can simultaneously work on different spots on the map. During the exercise, students may leave the setting at any time. Orphan cubes can then be used by other groups if necessary. County 

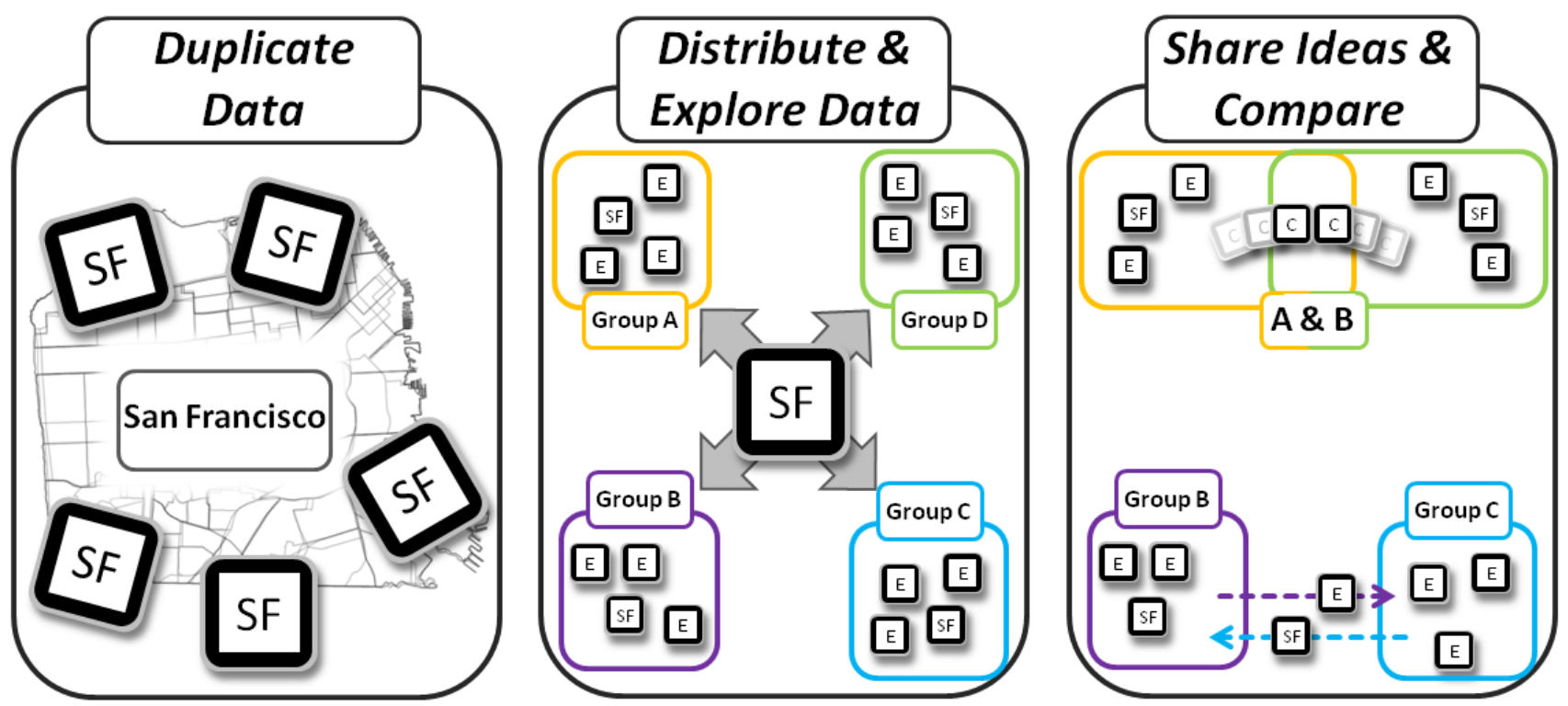

Figure 8. Storyline: The data is copied from the multi-touch table to the smart tangible devices. Therefore, several people can work with the same data without hindering themselves due to the limited space around the multi-touch table. For an in-depth analysis additional cubes are used to reveal detailed information. Finally the findings can be shared with others by exchanging and/or connecting data cubes.

information can be loaded on a data cube by placing the cube on top of a county and pressing the display. The same county can be associated with multiple cubes, allowing multiple groups investigating the same data independently. These groups can then move to nearby working environments without hinder the others in their analysis. In our approach users build up their exploration space by arranging cubes as needed, no explicit exploration space on the multi-touch table is required to be maintained by the system. The system does not need to track users but only the states and neighborhood of cubes, which enables our interface for scenarios with many users interacting with the data.

Figure 9 shows a small part of the setting. A user group interested in the county of San Francisco, has loaded its data point on a cube. The data cube then shows the same glyph for San Francisco than on the map. To get further details, the group extended the data cube with an exploration cube to the right, which provides more details about of the single dimensions. To get even more details, they are currently extending the data cube with a second exploration cube.

Looking at the glyph and the details, the group discovers an unexpected change in the domestic migration rate from 2011 to 2012. They figured out, that there is a high increase of the domestic migration in San Francisco till 2012, compared to the international migration rate, which remains stable. To compare the migration rate of different counties, cubes can be shared between groups. In our scenario, the student group assumes that the same migration behavior as seen in San Francisco can be found in neighboring counties. To share their knowledge, they simply hand over the data cube to other students investigating Contra Costa County. Surprisingly, the comparison of the counties shows them an unexpected result. The domestic and the international migration rate of Contra Costa County is stable for this time period. As a final step, the students pass their findings to the supervisor who verifies their finding.

\section{LIMITATIONS}

Although, our approach of a tangible exploration of multi-dimensional data points provides a playful and collaborative entry into an early analysis, it still has some limitations. The most restricting limitation is the small screen size and low resolution of the Sifteo Cubes with $128 \times 128$ pixels. It does not provide sufficient screen real estate to display richer visualizations that can offer additional perspectives on data points and further assist 


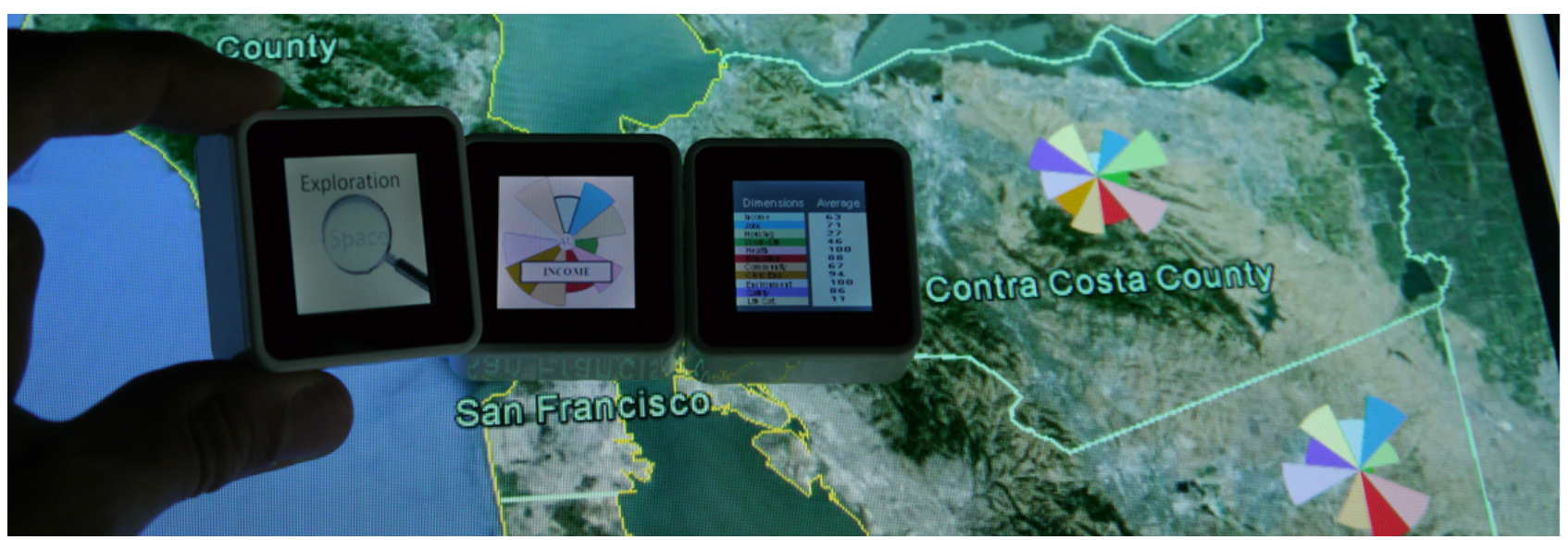

Figure 9. Use Case: Smart tangible devices are used to explore the data of San Francisco

users during decision making. However, it is foreseeable that upcoming technology will settle this by the progress of miniaturization of display devices and its accompanying increase of display resolution.

Moreover, the modular arrangement of cubes in physical space comes at cost of an automatic layouting of data points. At the moment, the arrangement of data points has to be done manually by the users. Nevertheless, recent research in HCI shows the technical feasibility of tangible user interfaces that demonstrate an automatic movement and positioning of physical objects on a flat horizontal surface utilizing small actuated ${ }^{\sqrt{50}}$ or motorized tangibles.

\section{FUTURE WORK}

Our basic concept using Sifteo Cubes in data analysis is not restricted to our presented use case. The flexibility and the interactive possibilities of this device offer many more challenging research areas.

\subsection{Information Stacking for Multi-Touch Tables}

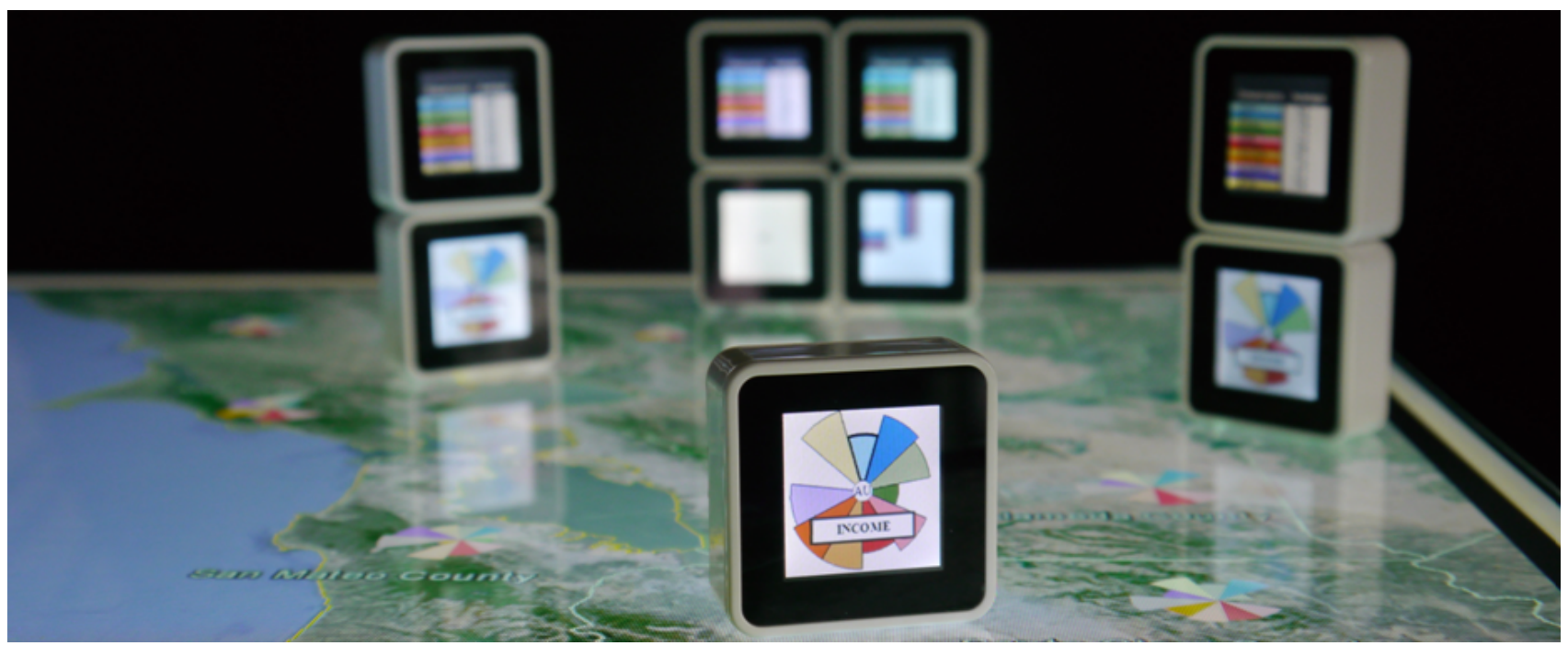

Figure 10. Information Stacking: Example setting of multiple information stacks on top of a multi-touch table. Depending on required granularity, details of data points can be visualized with multiple stacked cubes.

In combination with a multi-touch table designers can think of extending the 2 dimensional space with a third dimension by stacking the cubes on top of each other on the surface. An example prototype is shown in 
Figure 10. This adds additional screen real estate without loosing space on the multi-touch table. Besides the fact that this arrangement would reduce overlap by covering only a small area of the surface, there is another advantage facilitating the exploration. With information stacks analysts can start exploring multiple points of interest simultaneously without missing details about the context.

\subsection{Tangible File System}

An entirely different approach is the possibility of investigating Sifteo Cubes as a tangible file system representation. According to the arrangement of the cubes, the hierarchical order of a file system is displayed. Cubes can be layouted in a tree with a starting root node and several branches. By adding additional cubes the branches can be explored in more detail until the cubes represent real data points (e.g., pictures, movies, etc.). By touching a cube several times the user can switch between different interaction modes (copying, cutting, etc.). For a simple copy and paste command the user has to select the correct mode, take the cube with the data and put it to the destined location (e.g., in a different branch). Additionally, other users can easily copy their data to the initial file structure or take data with them. This intuitive data exchange is an interesting area when investigating tangible file systems and collaborative work.

\section{CONCLUSIONS}

We presented an interaction design for data analysis in collaborative group work. The design is multi-modal and supports mixed-focus collaboration. The application example shows the applicability and usefulness of our approach for real world analysis scenarios. A small qualitative user study helped us to create a thorough interaction concept, which can be easily understood by users without a computer science background. As a next step, we will conduct an experiment to investigate users working with the cubes to explore real data visually. The results will help us not only to improve our current interaction design based on Sifteo Cubes but to gain additional knowledge about the usefulness of smart tangible objects for interactive and collaborative visual data analysis. This information is mandatory when combining smart tangible objects with multi-touch tables. People should not be overwhelmed by the possibilities provided by the two devices.

Our approach is not necessarily restricted to a multi-touch table presenting a geographic maps. It is possible to extend any static visualization technique with smart tangibles. In this case, the visualization benefits from the collaborative design of our interaction technique and is able to be used in public spaces.

\section{ACKNOWLEDGMENTS}

This work has been supported by the Consensus project and has been partly funded by the European Commissions 7th Framework Programme through theme ICT-2013.5.4 ICT for Governance and Policy Modelling under contract no.611688.

\section{REFERENCES}

[1] "Your better life index," (2011). http://www.oecdbetterlifeindex.org/. Retrieved July 2013.

[2] Wigdor, D. and Wixon, D., [Brave NUI world: designing natural user interfaces for touch and gesture], Morgan Kaufmann (2011).

[3] Tang, A., Tory, M., Po, B., Neumann, P., and Carpendale, S., "Collaborative coupling over tabletop displays," in [In Proc. of CHI '06], 1181-1190, ACM Press, New York, New York, USA (Apr. 2006).

[4] Kirsh, D., "Thinking with external representations," AI 83 Society 25, 441-454 (Feb. 2010).

[5] Jacob, R. J., Girouard, A., Hirshfield, L. M., Horn, M. S., Shaer, O., Solovey, E. T., and Zigelbaum, J., "Reality-based interaction: a framework for post-WIMP interfaces," in [CHI '08: Proceeding of the twentysixth annual SIGCHI conference on Human factors in computing systems], 201-210, ACM, New York, NY, USA (2008).

[6] Merrill, D., Sun, E., and Kalanithi, J., "Sifteo cubes," in [Proceedings of the 2012 ACM annual conference extended abstracts on Human Factors in Computing Systems Extended Abstracts], CHI EA '12, 1015-1018, ACM, New York, NY, USA (2012). 
[7] Merrill, D., Kalanithi, J., and Maes, P., "Siftables: towards sensor network user interfaces," in [Proceedings of the 1st international conference on Tangible and embedded interaction], 75-78, ACM (2007).

[8] Du Toit, S. H., Steyn, A. G. W., and Stumpf, R. H., [Graphical exploratory data analysis], Springer-Verlag New York, Inc. (1986).

[9] Xu, P., Du, F., Cao, N., Shi, C., Zhou, H., and Qu, H., "Visual analysis of set relations in a graph," in [Computer Graphics Forum], 32(3pt1), 61-70, Wiley Online Library (2013).

[10] Yau, N., [Data Points: Visualization That Means Something], John Wiley \& Sons (2013).

[11] Tufte, E., [Envisioning information.], Graphics Press (1990).

[12] Brandes, U., Nick, B., Rockstroh, B., and Steffen, A., "Gestaltlines," in [Computer Graphics Forum], 32(3pt2), 171-180, Wiley Online Library (2013).

[13] Pickett, R. M. and Grinstein, G. G., "Iconographic displays for visualizing multidimensional data," in [Proceedings of the 1988 IEEE Conference on Systems, Man, and Cybernetics], 514, 519 (1988).

[14] Beddow, J., "Shape coding of multidimensional data on a microcomputer display," in [Visualization, 1990. Visualization'90., Proceedings of the First IEEE Conference on], 238-246, IEEE (1990).

[15] Borgo, R., Proctor, K., Chen, M., Janicke, H., Murray, T., and Thornton, I. M., "Evaluating the impact of task demands and block resolution on the effectiveness of pixel-based visualization," Visualization and Computer Graphics, IEEE Transactions on 16(6), 963-972 (2010).

[16] Abdul-Rahman, A., Lein, J., Coles, K., Maguire, E., Meyer, M., Wynne, M., Johnson, C., Trefethen, A., and Chen, M., "Rule-based visual mappings-with a case study on poetry visualization,"

[17] Keim, D. A. and Oelke, D., "Literature fingerprinting: A new method for visual literary analysis," in [Visual Analytics Science and Technology, 200\%. VAST 200\%. IEEE Symposium on], 115-122, IEEE (2007).

[18] Hartigan, J. A., "Printer graphics for clustering," Journal of Statistical Computation and Simulation 4(3), 187-213 (1975).

[19] Fischer, F., Fuchs, J., Vervier, P.-A., Mansmann, F., and Thonnard, O., "Vistracer: a visual analytics tool to investigate routing anomalies in traceroutes," in [Proceedings of the Ninth International Symposium on Visualization for Cyber Security], 80-87, ACM (2012).

[20] Cleveland, W. and McGill, R., "Graphical perception: Theory, experimentation, and application to the development of graphical methods," Journal of the American Statistical Association, 531-554 (1984).

[21] Chernoff, H., "The use of faces to represent points in k-dimensional space graphically," Journal of the American Statistical Association, 361-368 (1973).

[22] Siegel, J., Farrell, E., Goldwyn, R., and Friedman, H., "The surgical implications of physiologic patterns in

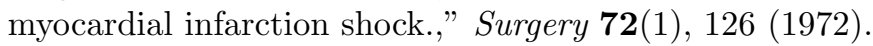

[23] Anderson, E., "A semigraphical method for the analysis of complex problems," Proceedings of the National Academy of Sciences of the United States of America 43(10), 923 (1957).

[24] Chambers, J. M., [Graphical methods for data analysis] (1983).

[25] Gnanadesikan, R., [Methods for statistical data analysis of multivariate observations], vol. 321, John Wiley \& Sons (2011).

[26] Tatu, A., Zhang, L., Bertini, E., Schreck, T., Keim, D., Bremm, S., and von Landesberger, T., "Clustnails: Visual analysis of subspace clusters," Tsinghua Science and Technology 17(4), 419-428 (2012).

[27] Levkowitz, H., "Color icons-merging color and texture perception for integrated visualization of multiple parameters," in [Visualization, 1991. Visualization'91, Proceedings., IEEE Conference on], 164-170, IEEE (1991).

[28] Chuah, M. C. and Eick, S. G., "Information rich glyphs for software management data," Computer Graphics and Applications, IEEE 18(4), 24-29 (1998).

[29] Kintzel, C., Fuchs, J., and Mansmann, F., "Monitoring large ip spaces with clockview," in [Proceedings of the 8th International Symposium on Visualization for Cyber Security], 2, ACM (2011).

[30] Fischer, F., Fuchs, J., and Mansmann, F., "Clockmap: Enhancing circular treemaps with temporal glyphs for time-series data," Proc. Euro Vis Short Papers, Eurographics , 97-101 (2012).

[31] Mezzich, J. E. and Worthington, D. R., "A comparison of graphical representations of multidimensional psychiatric diagnostic data," Graphical representation of multivariate data , 123-141 (1978). 
[32] Nightingale, F., [Notes on Matters Affecting the Health, Efficiency, and Hospital Administration of the British Army: Founded Chiefly on the Experience of the Late War. By Florence Nightingale. Presented by Request to the Secretary of State for War], Harrison and Sons, St. Martin's Lane, WC (1858).

[33] Pearlman, J., Rheingans, P., and des Jardins, M., "Visualizing diversity and depth over a set of objects," Computer Graphics and Applications, IEEE 27(5), 35-45 (2007).

[34] Chung, D. H., Legg, P. A., Parry, M. L., Bown, R., Griffiths, I. W., Laramee, R. S., and Chen, M., "Glyph sorting: Interactive visualization for multi-dimensional data," arXiv preprint arXiv:1304.2889 (2013).

[35] Fuchs, J., Fischer, F., Mansmann, F., Bertini, E., Isenberg, P., et al., "Evaluation of alternative glyph designs for time series data in a small multiple setting," in [Proceedings of the Conference on Human Factors in Computing Systems (CHI)], (2013).

[36] Ward, M., "Multivariate data glyphs: Principles and practice," Handbook of Data Visualization, 179-198 (2008).

[37] Borgo, R., Kehrer, J., Chung, D. H., Maguire, E., Laramee, R. S., Hauser, H., Ward, M., and Chen, M., "Glyph-based visualization: Foundations, design guidelines, techniques and applications," in [Eurographics 2013-State of the Art Reports], 39-63, The Eurographics Association (2012).

[38] Fitzmaurice, G. W., Ishii, H., and Buxton, W. A. S., "Bricks: laying the foundations for graspable user interfaces," in [In Proc of CHI '95], 442-449, ACM Press, New York, NY, USA (May 1995).

[39] Baudisch, P., Becker, T., and Rudeck, F., "Lumino," in [Proceedings of the 28th international conference on Human factors in computing systems - CHI '10], 1165, ACM Press, New York, New York, USA (Apr. 2010).

[40] Leitner, J. and Haller, M., "Geckos," in [Proceedings of the 2011 annual conference on Human factors in computing systems - CHI'11], 2985, ACM Press, New York, New York, USA (May 2011).

[41] Hennecke, F., Wimmer, R., Vodicka, E., and Butz, A., "Vertibles," in [Proceedings of the Sixth International Conference on Tangible, Embedded and Embodied Interaction - TEI '12], 303, ACM Press, New York, New York, USA (Feb. 2012).

[42] Rekimoto, J., Ullmer, B., and Oba, H., "DataTiles," in [Proceedings of the SIGCHI conference on Human factors in computing systems - CHI '01], 269-276, ACM Press, New York, New York, USA (Mar. 2001).

[43] Spindler, M., Tominski, C., Schumann, H., and Dachselt, R., "Tangible views for information visualization," in [ACM International Conference on Interactive Tabletops and Surfaces], 157-166, ACM (2010).

[44] Klum, S., Isenberg, P., Langner, R., Fekete, J., and Dachselt, R., "Stackables: combining tangibles for faceted browsing," in [Proceedings of the International Working Conference on Advanced Visual Interfaces], 241-248, ACM (2012).

[45] Zigelbaum, J., Horn, M. S., Shaer, O., and Jacob, R. J. K., "The tangible video editor," in [In Proc. of TEI '07], 43-46, ACM Press, New York, New York, USA (Feb. 2007).

[46] Bertin, J., [Semiology of Graphics: Diagrams, Networks, Maps, trans] (1983).

[47] Ware, C., [Information visualization: perception for design], Morgan Kaufmann (2012).

[48] Ekman, G. and Junge, K., "Psychophysical relations in visual perception of length, area and volume," Scandinavian Journal of Psychology 2(1), 1-10 (1961).

[49] Andrienko, N. and Andrienko, G., [Exploratory Analysis of Spatial and Temporal Data: A Systematic Approach], Springer (2006).

[50] Weiss, M., Schwarz, F., Jakubowski, S., and Borchers, J., "Madgets: Actuating Widgets on Interactive Tabletops," in [In Proc of UIST '10], 293-302, ACM Press, New York, New York, USA (Oct. 2010).

[51] Pedersen, E. W. and Hornbæk, K., "Tangible bots," in [In Proc. of CHI '11], 2975, ACM Press, New York, New York, USA (May 2011). 